\title{
Decomposition of the neutron reflection coefficient in the stratified surface layer model
}

\author{
Loren R. Clifford ${ }^{1}$ \\ Department of Nuclear Engineering, The University of Michigan. Ann Arbor, MI 48109, USA
}

\begin{abstract}
A neutron reflection coefficient can be decomposed into two simpler reflection coefficients: the Fresnel coefficient for bulk material only, and a coefficient for the case where the surface layer is surrounded on both sides by bulk material. The latter coefficient is obtained via the usual matrix product whose factors correspond to homogeneous strata of the surface layer; or this coefficient can also be expressed as a sum of terms, one for each stratum of the surface layer. The purpose of this latter theorem is to reveal the algebraic structure of the scattering problem and the relation of the matrix method to the Born approximation. Zero absorption and diffuse scattering is assumed.
\end{abstract}

\section{Introduction}

Two ways to calculate the neutron reflection coefficient for a planar stratified sample use the Riccati equation [1] and the matrix method [2], respectively. Let the air-sample interface be the plane surface $z=0$ with positive $z$-axis projecting into the sample. The neutron wave is at glancing incidence outside the reflecting medium $(z<0)$. It has a $z$-component

$\Psi(z)=\mathrm{e}^{\mathrm{i} k_{z} z}+R \mathrm{e}^{-\mathrm{i} k_{z} z}$,

where $R$ is the reflection coefficient, which depends upon $k_{z}$. Inside the medium the local propagation number $k(z)$ is given by the equation:

$k^{2}(z)=k_{z}^{2}-4 \pi \rho(z) \quad(z>0)$,

where $\rho(z)$ is the average scattering length density. In the stratified model the sample consists of layers

\footnotetext{
${ }^{1}$ Also at: Department of Physics, Central Michigan University, Mount Pleasant, MI 48859, USA
}

with propagation numbers $k_{\alpha}$, given by Eq. (2), of variable thicknesses $d_{\alpha}$, where $\alpha=1,2, \ldots, N$. The layer closest to the air-sample interface is labeled $\alpha=1$ and that adjacent to the bulk part of the medium is labeled $\alpha=N$. The bulk part of the sample is assumed to be semi-infinite so that no reflected wave exists there, i.e.

$\Psi \propto \mathrm{e}^{\mathrm{i} q z} \quad$ (bulk material)

with $q$ defined by the equation

$q^{2}=k_{z}^{2}-4 \pi \rho$ (bulk).

The appropriate root of Eq. (4) puts $q$ either on the positive real axis or on the positive imaginary axis. In practice the scattering length density of the bulk material is known so that $q$ is also known.

\section{Riccati equation}

Only the logarithmic derivative,

$\xi=\frac{1}{\Psi} \frac{\mathrm{d} \Psi}{\mathrm{d} z}$ 
evaluated at $z=0$ is needed to find the reflection coefficient. From Eq. (1) we have

$\Psi(0)=1+R$,

$\frac{\mathrm{d} \Psi}{\mathrm{d} z}(0)=\mathrm{i} k_{z}(1-R)$

so that the reflection coefficient is

$R=\frac{i k_{z}-\xi(0)}{\mathrm{i} k_{z}+\xi(0)}$

The logarithmic derivative obeys the first-order Riccati equation

$\frac{\mathrm{d} \xi}{\mathrm{d} z}+\xi^{2}+k^{2}=0$

whose solution is determined by the single boundary condition

$\xi(\mathrm{bulk})=\mathrm{i} q$

Although Eq. (9) is nonlinear, it is easily integrated in the stratified model, and even if $\rho(z)$ is discontinuous, $\xi(z)$ is continuous and $\xi(0)$ is unambiguous.

\section{Matrix method}

The matrix method and the decomposition procedure to be presented involve a function $M$ of $k_{z}$ which is related to $\xi(0)$ by the equation:

$\xi(0)=\mathrm{i} q \frac{1-M}{1+M}$

The reflection coefficient (8) becomes

$R=\frac{F+M}{1+F M}$

where $F$ is the Fresnel coefficient,

$F=\frac{k_{z}-q}{k_{z}+q}$, corresponding to the case of bulk material only. The function $M$ is also a reflection coefficient which corresponds to the case when $k_{z}=q$, i.e. a situation in which the sample (surface and bulk material) remains unchanged, but the air (incident medium) is replaced with bulk material so that the wave is incident with $k_{z}$ equal to $q$. Then $F$ is zero and $R$ is equal to $M$. If $q$ is pure imaginary, then $M$ is real (see Appendix A) and $R$ has unit modulus which is the case of total reflection.

Because $M$ is a reflection coefficient it can be generated in the stratified model by the matrix method. The equivalence between the matrix method shown here and that given by Lekner is demonstrated in Appendix B. Introduce a $2 \times 2$ matrix,

$O_{\alpha}=\left(\begin{array}{ll}\cos \left(k_{\alpha} d_{\alpha}\right) & \frac{q}{k_{\alpha}} \sin \left(k_{\alpha} d_{\alpha}\right) \\ -\frac{k_{\alpha}}{q} \sin \left(k_{\alpha} d_{\alpha}\right) & \cos \left(k_{\alpha} d_{\alpha}\right)\end{array}\right)$,

for the $\alpha$ th stratum of thickness $d_{\alpha}$ and propagation number $k_{\alpha}$, and define $P_{\alpha}$ and $Q_{\alpha}$ by the matrix product

$\left(\begin{array}{c}P_{\alpha} \\ Q_{\alpha}\end{array}\right)=O_{1} O_{2} O_{3} \cdots O_{\alpha-1} O_{\alpha}\left(\begin{array}{c}P_{0} \\ Q_{0}\end{array}\right)$

with starting values $P_{0}=1 / 2$ and $Q_{0}=-\mathrm{i} / 2$. Then $M$ is given by

$M=\frac{P_{N}-\mathrm{i} Q_{N}}{P_{N}+\mathrm{i} Q_{N}}$,

where $\alpha=N$ denotes the stratum adjacent to the bulk material. This result together with Eq. (12) gives the reflection coefficient $R$ in closed form.

\section{Decomposition of $M$}

It is possible to expand $M$ into a series of terms, one for each stratum of the surface layer. Define the quantities

$S_{\alpha}=P_{\alpha}+\mathrm{i} Q_{\alpha}$ 
for $\alpha=0,1,2, \ldots, N$. The procedure for generating the entire series is illustrated by the first steps. Write

$M=1-\frac{2 \mathrm{i} Q_{N}}{S_{N}}=\frac{S_{N-1}}{S_{N-1}}-\frac{2 \mathrm{i} Q_{N} S_{N-1}}{S_{N-1} S_{N}}$

and then put this into the form

$M=\frac{P_{N-1}-\mathrm{i} Q_{N-1}}{P_{N-1}+\mathrm{i} Q_{N-1}}+\frac{2 \mathrm{i}\left[Q_{N-1} S_{N}-Q_{N} S_{N-1}\right]}{S_{N-1} S_{N}}$

Repeat the steps on the first term of Eq. (19). The second term is simplified using

$2 \mathrm{i}\left[Q_{\alpha-1} S_{\alpha}-Q_{\alpha} S_{\alpha-1}\right]=\frac{q^{2}-k_{\alpha}^{2}}{2 \mathrm{i} q k_{\alpha}} \sin \left(k_{\alpha} d_{\alpha}\right)$,

which depends upon the fact that the matrices $O_{\alpha}$ have unit determinant (Appendix C). Note that $q^{2}-k_{\alpha}^{2}$ is independent of $k_{z}$. If the scattering length density of the $\alpha$ th stratum is $\rho_{\alpha}$, then this difference is

$U_{\alpha}=q^{2}-k_{\alpha}^{2}=4 \pi\left[\rho_{\alpha}-\rho(\right.$ bulk $\left.)\right]$.

The process outlined in Eqs. (19) and (20) is repeated $N-1$ times to generate the decomposition of $M$ into a series of terms

$M=\sum_{\alpha=1}^{N} M_{\alpha}$.

The term for the $\alpha$ th stratum of the surface layer is

$M_{\alpha}=\frac{q^{2}-k_{\alpha}^{2}}{2 \mathrm{i} q k_{\alpha}} \frac{\sin \left(k_{\alpha} d_{\alpha}\right)}{S_{\alpha-1} S_{\alpha}}$.

The series (22) is self-terminating because $M_{\alpha}$ vanishes when $k_{\alpha}=q$, i.e. when the bulk material is reached.

The terms of the series (22) are most simple for strata close to the air-sample interface. In $M_{1}$ the denominator contains $S_{0}=1$ and (putting all thicknesses $d_{\alpha}=\Delta z$ for simplicity)

$$
\begin{aligned}
S_{1}=\frac{1}{4 k_{1} q}\left[\left(q-k_{1}\right)\left(k_{1}-q\right) \mathrm{e}^{\mathrm{i} k_{1} \Delta z}\right. & \\
& \left.+\left(q+k_{1}\right)\left(k_{1}+q\right) \mathrm{e}^{-\mathrm{i} k_{1} \Delta z}\right] .
\end{aligned}
$$

The denominator of $M_{2}$ contains $S_{1}$ and

$$
\begin{aligned}
S_{2}= & \frac{1}{8 k_{1} k_{2} q}\left[\left(q-k_{2}\right)\left(k_{2}+k_{1}\right)\left(k_{1}-q\right) \mathrm{e}^{\mathrm{i}\left(k_{2}+k_{1}\right) \Delta z}\right. \\
& +\left(q-k_{2}\right)\left(k_{2}-k_{1}\right)\left(k_{1}+q\right) \mathrm{e}^{\mathrm{i}\left(k_{2}-k_{1}\right) \Delta z} \\
& +\left(q+k_{2}\right)\left(k_{2}-k_{1}\right)\left(k_{1}-q\right) \mathrm{e}^{-\mathrm{i}\left(k_{2}-k_{1}\right) \Delta z} \\
& \left.+\left(q+k_{2}\right)\left(k_{2}+k_{1}\right)\left(k_{1}+q\right) \mathrm{e}^{-\mathrm{i}\left(k_{2}+k_{1}\right) \Delta z}\right] .
\end{aligned}
$$

\section{The Born approximation}

In each $S_{\alpha}$ there is one term, written as the last term in $S_{1}$ and $S_{2}$, which has only plus signs between the propagation numbers, and it is this term that gives the Born approximation. When $k_{z}^{2}$ is large compared with $4 \pi \rho(z)$ (the Born approximation limit), $q$ and all the $k_{\alpha}$ 's approach the value $k_{z}$ and only the term in $S_{\alpha}$ with all plus signs remains. Thus in the Born approximation we have

$S_{\alpha} \approx \mathrm{e}^{-\mathrm{i} \alpha k_{\mathrm{z}} \Delta z}$.

The Born approximation expression for $M_{\alpha}$ is

$M_{\alpha} \approx \frac{U_{\alpha}}{2 \mathrm{i} k_{z}^{2}} \mathrm{e}^{\mathrm{i}(2 \alpha-1) k_{z} \Delta z} \sin \left(k_{z} \Delta z\right)$,

which can be put into the more recognizable form,

$M_{\alpha} \approx \frac{U_{\alpha}}{2 \mathrm{i} k_{z}} \int_{(\alpha-1) \Delta z}^{\alpha \Delta z} \mathrm{e}^{2 \mathrm{i} k_{z} z} \mathrm{~d} z$.

To see that this is the correct Born approximation expression for $M_{\alpha}$ note that $M$ can also be regarded 
as the reflection coefficient for a sample consisting only of a surface layer with air on both sides. The scattering length density in this surface layer is $\rho(z)-\rho$ (bulk) in place of $\rho(z)$ and the incident propagation number is $q$ instead of $k_{z}$.

It is often pointed out that in the Born approximation, where the reflection coefficient is a Fourier transform of the scattering length density, the reflectivity (modulus of the reflection coefficient squared) for a sample of finite thickness remains unchanged when the sample is reversed, or equivalently when the wave is incident on the other side of the sample [3]. The reflection coefficient $M$ represents this kind of situation insofar as it corresponds to a sample of finite thickness. If $M_{r}$ differs from $M$ only by reversal of the sample (surface layer), then Eq. (28) can be used to obtain the usual result

$M_{r}=M^{*}$ times a phase factor,

in the Born approximation (* denotes complex conjugate). The same conclusion turns out to be true when $M$ is computed exactly in the stratified model. This can be easily proven with the help of Eqs. (B1) and (B3) of Appendix B which represent a sample of finite thickness and the same sample with strata in reverse order, respectively.

\section{Acknowledgement}

I would like to thank Professor George C. Summerfield of the Department of Nuclear Engineering, The University of Michigan, for his guidance and encouragement during this work.

\section{Appendix A}

To show that $M$ is real when $q$ is purely imaginary $\left(k_{z}^{2}<4 \pi \rho\right.$ (bulk)), consider the matrices (14) which have only real elements when $q$ is real regardless of whether $k_{\alpha}$ is real or purely imaginary. However, if $q$ is purely imaginary the upper right and lower left elements of every $O_{\alpha}$ matrix are purely imaginary. When $O_{N}$ acts on the starting vector with components $P_{0}=1 / 2$ and $Q_{0}=-\mathrm{i} / 2$ in this latter case, the result is a new vector whose upper component is again real and whose lower component is again purely imaginary. Similarly, when $O_{N-1}$ operates on this new vector, and $q$ is purely imaginary, the result is the same, i.e. upper component of the resulting vector is real, lower component purely imaginary. The final result when $q$ is purely imaginary is that $P_{N}$ is real and $Q_{N}$ is purely imaginary so that $M$, as given by (16), is real.

It is a striking fact that whenever $q$ is real (reflection less than total) the entire matrix,

$O=O_{1} O_{2} O_{3} \cdots O_{N-1} O_{N}$

has all real components but $M$, as given by Eq. (16), is a complex function of $k_{z}$.

\section{Appendix B}

Lekner uses a matrix

$O_{N} O_{N-1} \cdots O_{3} O_{2} O_{1}=\left(\begin{array}{ll}m_{11} & q m_{12} \\ \frac{1}{q} m_{21} & m_{22}\end{array}\right)$.

The inverse matrix,

$$
\begin{gathered}
O_{1}^{-1} O_{2}^{-1} O_{3}^{-1} \cdots O_{N-1}^{-1} O_{N}^{-1} \\
=\left(\begin{array}{ll}
m_{22} & -q m_{12} \\
-\frac{1}{q} m_{21} & m_{11}
\end{array}\right)
\end{gathered}
$$

can be analyzed in terms of the individual $O_{\alpha}$ matrices which have corresponding inverses, i.e. the diagonal elements (which are equal for the $O_{\alpha}$ matrices) exchange places and the off-diagonal elements change sign; in other words $O_{\alpha}^{-1}$ is obtained from $O_{\alpha}$ by changing the sign of $q$. It follows that the reversed matrix product is

$O_{1} O_{2} O_{3} \cdots O_{N-1} O_{N}=\left(\begin{array}{ll}m_{22} & q m_{12} \\ \frac{1}{q} m_{21} & m_{11}\end{array}\right)$

which differs from Eq. (B1) in that $m_{11}$ and $m_{22}$ are interchanged. 
Aside from a translational phase factor, Lekner's reflectivity expression (Eq. (46) of Ref. [2]) is

$R=\frac{q k_{z} m_{12}+\mathrm{i} k_{z} m_{22}-\mathrm{i} q m_{11}+m_{21}}{q k_{z} m_{12}+\mathrm{i} k_{z} m_{22}+\mathrm{i} q m_{11}-m_{21}}$.

From Eq. (B3) we see that

$P_{N}=\frac{1}{2}\left(m_{22}-\mathrm{i} q m_{12}\right)$,

$Q_{N}=\frac{1}{2}\left(\frac{1}{q} m_{21}-\mathrm{i} m_{11}\right)$,

and Eq. (B4) can be written as

$R=\frac{k_{z} P_{N}-\mathrm{i} q Q_{N}}{k_{z} P_{N}+\mathrm{i} q Q_{N}}$

which is equivalent to Eq. (12) with $M$ given by Eq. (16). It was necessary to reverse the order of the matrix multiplication, i.e. to use Eq. (B3) instead of Eq. (B1) in order to make the decomposition procedure Eqs. (18)-(20) possible.

\section{Appendix C}

In order to establish Eq. (20) write

$O_{1} O_{2} \cdots O_{\alpha-1}=\left(\begin{array}{ll}O_{11} & O_{12} \\ O_{21} & O_{22}\end{array}\right)$

so that Eq. (15) becomes

$$
\begin{aligned}
& P_{\alpha}=O_{11}\left[\frac{1}{2} \cos \left(k_{\alpha} d_{\alpha}\right)-\frac{\mathrm{i}}{2} \frac{q}{k_{\alpha}} \sin \left(k_{\alpha} d_{\alpha}\right)\right] \\
& +O_{12}\left[-\frac{1}{2} \frac{k_{\alpha}}{q} \sin \left(k_{\alpha} d_{\alpha}\right)-\frac{\mathrm{i}}{2} \cos \left(k_{\alpha} d_{\alpha}\right)\right],
\end{aligned}
$$

$$
\begin{aligned}
Q_{\alpha}= & O_{21}\left[\frac{1}{2} \cos \left(k_{\alpha} d_{\alpha}\right)-\frac{\mathrm{i}}{2} \frac{q}{k_{\alpha}} \sin \left(k_{\alpha} d_{\alpha}\right)\right] \\
& +O_{22}\left[-\frac{1}{2} \frac{k_{\alpha}}{q} \sin \left(k_{\alpha} d_{\alpha}\right)-\frac{\mathrm{i}}{2} \cos \left(k_{\alpha} d_{\alpha}\right)\right] .
\end{aligned}
$$

Furthermore, we have

$$
\begin{aligned}
& P_{\alpha-1}=\frac{1}{2} O_{11}-\frac{\mathrm{i}}{2} O_{12}, \\
& Q_{\alpha-1}=\frac{1}{2} O_{21}-\frac{\mathrm{i}}{2} O_{22} .
\end{aligned}
$$

Using definition (17) of $S_{\alpha}$ we find that

$$
\begin{aligned}
2 \mathrm{i} & {\left[Q_{\alpha-1} S_{\alpha}-Q_{\alpha} S_{\alpha-1}\right] } \\
& =\frac{\mathrm{i}}{2}\left[O_{11} O_{22}-O_{12} O_{21}\right]\left(\frac{k_{\alpha}}{q}-\frac{q}{k_{\alpha}}\right) \sin \left(k_{\alpha} d_{\alpha}\right) .
\end{aligned}
$$

The proof of Eq. (20) is completed by noting that the matrix (C1) has unit determinant since all of its constituent matrices have unit determinant.

\section{References}

[1] K. Chadan and P.C. Sabatier, Inverse Problems in Quantum Scattering Theory (Springer, Berlin, 1989; 2nd ed.) p. 421.

[2] John Lekner,Physica B 173 (1991) 107.

[3] D.S. Sivia, W.A. Hamilton and G.S. Smith, Physica B 173 (1991) 123. 